\title{
Propuesta para la identificación de grupos de interés y sus legítimas demandas desde los derechos humanos en pymes de México
}

\author{
JUAN ALBERTO AMÉZQUITA-ZAMORA ${ }^{a}$, \\ JOSÉ CARLOS VÁZQUEZ-PARRA ${ }^{b}$, \\ FLORINA GUADALUPE ARREDONDO-TRAPERO ${ }^{c}$
}

RESUMEN Uno de los mayores retos de las empresas contemporáneas es ser productivas y competentes, pero a su vez, responder a las demandas legítimas que la sociedad y sus grupos de interés les exigen. Si bien la protección y no violentación de los derechos humanos se constituye en una de estas obligaciones sociales, con base en parámetros del Pacto Mundial y los Principios Rectores para la Empresas y los Derechos Humanos, el ser respetuoso no es suficiente. El presente artículo busca responder a esta situación, al plantear la necesidad de adoptar un modelo de identificación de los grupos de interés desde una perspectiva de derechos humanos que apueste a una mejor integración de las necesidades y demandas de estos grupos, para la adopción de acciones que no solo respeten, sino que también promuevan y busquen el desarrollo de los derechos de los grupos de interés. La relevancia de esta propuesta se argumenta en el análisis de datos arrojados por los reportes de pymes mexicanas firmantes del Pacto Mundial, con base en los reportes de progreso que dichas organizaciones han realizado a partir de su adhesión al pacto.

\section{HISTORIA DEL ARTÍCULO}

¿CÓMO CITAR?:

Amézquita-Zamora, J.A., VázquezParra,J.C. \& Arredondo-Trapero, F.G. (2017). Propuesta para la identificación de grupos de interés y sus legítimas demandas desde los derechos humanos en pymes de México. Perspectiva Empresarial, 4(2), 47-58. http://dx.doi. org/10.16967/rpe.v4n2a5

RECIBIDO: 28 de junio de 2017 APROBADO: 16 de agosto de 2017

CORRESPONDENCIA: José Carlos Vázquez Parra, 3314298387. Av. General Ramón Corona 2514, Nuevo México, 45201 Zapopan, Jal., México.

PALABRAS CLAVE ética organizacional, responsabilidad social, stakeholders.

a Doctor, profesor de la Escuela de humanidades y educación del Tecnológico de Monterrey - Guadalajara, México. Correo electrónico: ja.amezquita@itesm.mx

b Doctor, profesor de la Escuela de humanidades y educación del Tecnológico de Monterrey - Guadalajara, México. Correo electrónico: jcvazquezp@itesm.mx

Doctora, profesor de la Escuela de humanidades y educación del Tecnológico de Monterrey - Monterrey, México. Correo electrónico: farredon@itesm.mx 
¿CóMO CITO EL ARTículO? HOW TO CITE THIS PAPER?

CHICAGO:

Amézquita-Zamora, Juan Alberto, Vázquez-Parra, José Carlos y ArredondoTrapero, Florina Guadalupe. 2017. "Propuesta para la identificación de grupos de interés y sus legítimas demandas desde los derechos humanos en pymes de México". Perspectiva Empresarial 4(2): 47-58. http://dx.doi. org/10.16967/rpe.v4n2a5

MLA:

Amézquita-Zamora, Juan Alberto, Vázquez-Parra, José Carlos y Arredondo-Trapero, Florina Guadalupe. "Propuesta para la identificación de grupos de interés y sus legítimas demandas desde los derechos humanos en pymes de México". Perspectiva Empresarial 4.2 (2017): 47-58. Digital. http:// dx.doi.org/10.16967/rpe. v4n2a5

\section{Proposal for the identification of stakeholders and their legitimate human rights claims in SMEs in Mexico}

ABSTRACT One of the greatest challenges for contemporary companies is to be productive and competent, while responding to the legitimate claims that society and its stakeholders make. Although the protection and non-violation of human rights constitutes one of these social obligations, based on parameters of the Global Compact and the Guiding Principles for Business and Human Rights, respectfulness is not enough. The present article intends to deal with this situation by suggesting the need to adopt a model for identifying stakeholders from a human rights perspective that is committed to a better integration of their needs and claims, and to take actions which not only respect, but also promote and seek the development of the rights of stakeholders. The relevance of this proposal is supported by the analysis of data from the progress reports prepared by Mexican SMEs who have joined the Global Compact.

KEYWORDS organizational ethics, social responsibility, stakeholders.

\section{Proposta para a identificação de grupos de interesse e suas legítimas demandas a partir dos direitos humanos em PMEs do México}

RESUMO Produtividade e competência, esses são os principais desafios das empresas contemporâneas, mas ao mesmo tempo, responder às demandas legítimas que a sociedade e seus grupos de interesse exigem. Ainda que a proteção e não violação dos direitos humanos sejam estabelecidas como uma dessas obrigações sociais, com base em parâmetros do Pacto Mundial e dos Princípios Orientadores sobre Empresas e Direitos Humanos, respeitar não é suficiente. O presente artigo busca responder a essa situação, ao propor a necessidade de adotar um modelo de identificação dos grupos de interesse a partir de uma perspectiva de direitos humanos que aposte em uma melhor integração das necessidades e demandas desses grupos, para a adoção de ações que não só respeitem, senão que também promovam e busquem o desenvolvimento dos direitos dos grupos de interesse. A relevância dessa proposta é argumentada na análise de dados lançados pelos informes de PMEs mexicanas que assinaram o Pacto Mundial, com base nos relatórios de progresso que essas organizações realizaram a partir da sua adesão ao pacto.

PALAVRAS CHAVE ética organizacional, responsabilidade social, stakeholders. 


\section{Introducción}

Hoy en día, la globalización parece ser el sello particular de lo que va del presente siglo, el cual se manifiesta en las organizaciones a partir de un entorno de alta competitividad, de constantes cambios en los ritmos de producción, así como de un ambiente institucional con colaboradores más competentes y consumidores más exigentes. Todo lo anterior es lo que obliga a que las empresas integren diversos y novedosos enfoques y perspectivas dentro de sus políticas, con la pretensión de alcanzar una mayor permanencia e integración en el mercado, y dar así una mejor respuesta a las demandas de la sociedad y de sus grupos de interés (Peláez, 2015). Propuestas internacionales como el Pacto Mundial o los Objetivos de Desarrollo Sostenible, han puesto especial atención en la necesidad de un compromiso organizacional hacia la resolución de problemas sociales, por lo que las empresas de hoy tienen que considerar muchas más cosas que sus antecesoras (Mota, Castelo \& da Silva, 2015).

Así, los planes de afrontamiento del nuevo fenómeno globalizador van más allá de gestar acciones concretas, como se venía haciendo en algunos modelos de responsabilidad social o filantropía corporativa, de manera que llegan a influir en las decisiones y la razón de ser de la propia empresa (Cantú, 2013). Esto hace que elementos tales como la cultura organizacional y la gobernanza interna se vuelvan piezas claves para responder a las nuevas necesidades del entorno, ya que estas se constituyen en la guía de acción que delimita los comportamientos, la interacción y las elecciones que la organización toma con respecto a los grupos de interés (Calderón, Murillo \& Torres, 2003). Sin embargo, ¿cómo identificar aquellos elementos que realmente resultan ser demandas legítimas de los grupos de interés propios de la empresa? ¿Cómo diferenciar estas obligaciones organizacionales, de aquellas acciones de responsabilidad social que no impactan verdaderamente a los agentes organizacionales? ¿Qué tan relevante resulta esto para las pymes mexicanas?

El presente artículo busca responder a estos cuestionamientos, al plantear la necesidad de adoptar un modelo de identificación de los grupos de interés desde una perspectiva de derechos humanos que apueste a una mejor integración de las necesidades y demandas de estos grupos, dentro de un modelo de negocios responsable, flexible e integral. La relevancia de esta propuesta se sustenta en el análisis de datos arrojados por los reportes de pymes mexicanas firmantes del Pacto Mundial, con base en los reportes de progreso que dichas organizaciones han realizado a partir de su adhesión al pacto. Se ha considerado como herramienta de análisis el Pacto Mundial, por ser uno de los esfuerzos internacionales más relevantes y representativos en cuanto a la participación de las empresas en los retos propuestos por la ONU en los Objetivos de Desarrollo Sostenible, ya que engloba temas de derechos humanos, protección del ambiente y políticas anticorrupción.

\section{Marco teórico}

\section{Los derechos humanos como obligación social de las empresas contemporáneas}

Durante el Foro Económico Mundial en Davos, celebrado en el 2016, Ban Ki-moon, exsecretario de la Organización de las Naciones Unidas (ONU), tuvo la oportunidad de dirigirse a los líderes del sector privado y plantearles la necesidad de un compromiso a nivel internacional para la construcción de un mundo más equitativo y respetuoso del medio ambiente y los derechos humanos (Ki-moon, 2016). Este señalamiento se apega claramente con lo pretendido a partir del Pacto Mundial, iniciativa de sostenibilidad corporativa de impacto internacional gestada en el marco de desarrollo de los Objetivos de Desarrollo del Milenio (Urteaga, 2008). Este pacto es un llamado a las empresas a alinear sus estrategias y operaciones con los principios universales de derechos humanos, estándares laborales, el medio ambiente y la lucha contra la corrupción, así como a llevar a cabo todas las acciones necesarias que permitan avanzar en los Objetivos de Desarrollo Sostenible (UNGlobal-Compact, 2015).

Las empresas que se unen al Pacto Mundial deben buscar desarrollar prácticas empresariales que se apeguen a los siguientes principios (Pacto-Mundial, 2015): 1. Apoyo, respeto y protección de los derechos humanos dentro de su ámbito de influencia; 2 . Asegurar que sus actividades no son cómplices de la vulneración de los derechos humanos; 3 . Apoyo a la libertad de asociación y el reconocimiento efectivo del derecho a 
la negociación colectiva; 4. Apoyo a la eliminación de toda forma de trabajo forzoso o realizado bajo coacción; 5. Apoyo a la erradicación del trabajo infantil; 6. Apoyo a la abolición de las prácticas de discriminación en el empleo y ocupación; 7. Mantenimiento de un enfoque preventivo que favorezca el medio ambiente; 8 . Fomento de iniciativas que promuevan una mayor responsabilidad ambiental; 9. Favorecer el desarrollo y la difusión de las tecnologías respetuosas en el medio ambiente; y 10. Trabajar contra la corrupción en todas sus formas, incluidas la extorsión y el soborno.

Bajo este decálogo, el Pacto Mundial fomenta una evolución en la visión de las empresas hacia los temas sociales, en la que la responsabilidad social y el fortalecimiento de los derechos humanos se constituyen en una parte fundamental de la sostenibilidad empresarial, integrándose en las operaciones y procesos cotidianos de la organización, así como en sus objetivos, misión y visión institucional (Pedroza, 2007). En palabras de Gustavo Pérez, presidente del Pacto Mundial México, el proyecto es buscar que las empresas ya no consideren la responsabilidad social y el cuidado de los derechos humanos como un gasto, como llegaba a pasar comúnmente con la filantropía, sino más bien, como una inversión y una parte natural de su organización, como sucede con los modelos de negocios responsables (Trejo, 2015). Sin embargo, esta no es la única iniciativa de este tipo, ya que en el 2011, el Consejo de Derechos Humanos de la ONU aprobó por consenso los Principios Rectores para las Empresas y los Derechos Humanos, los cuales se configuran como la principal aportación que la ONU ha generado sobre temas de empresa y derechos humanos. Estos principios ponen en práctica el marco de "Proteger, Respetar y Remediar", que la misma ONU aprobó durante el 2008.

Para la propuesta que aquí se plantea, se pretende que, de manera adicional a los principios del Pacto Mundial relacionados con los derechos humanos, las empresas se adhieran a lo estipulado por el "Principio 15" de los Principios Rectores, el cual señala:

Para cumplir con su responsabilidad de respetar los derechos humanos, las empresas deben contar con políticas y procedimientos apropiados en función de su tamaño y circunstancias. A saber:

a) Un compromiso político de asumir su responsabilidad de respetar los derechos humanos. b) Un proceso de diligencia debida en materia de derechos humanos para identificar, prevenir, mitigar y rendir cuentas de cómo abordan su impacto sobre los derechos humanos.

c) Unos procesos que permitan reparar todas las consecuencias negativas sobre los derechos humanos que hayan provocado o contribuido a provocar. (Ruggie, 2011, p. 17).

La primera exigencia, el compromiso político de respetar los derechos humanos, debe estar presente, por lo menos, en el código de conducta de la empresa, aunque lo ideal sería que fuera incorporado adecuadamente en la misión o la visión (Alfonso, 2013). La siguiente exigencia del "Principio 15" establece que las empresas deben emprender un proceso de debida diligencia, con la finalidad de determinar cuáles son los impactos sobre los derechos humanos, reales y potenciales, que pueden tener en su operación, así como las medidas que deben tomar a fin de prevenir los abusos y cómo han de rendir cuentas de los que se cometen en realidad. La última exigencia implica la obligación de establecer mecanismos y procesos que garanticen la reparación de las violaciones a los derechos humanos, lo cual requiere "el establecimiento de políticas explícitas e integrales" (Vives, 2014, p. 35), y la determinación, por parte de la directiva, de implementarlas.

El proceso que permita a las empresas cumplir con las exigencias establecidas en estos principios rectores, así como con el compromiso realizado con el Pacto Mundial, en lo que se refiere al deber de no violar los derechos humanos, suele ser muy arduo, lo cual puede inferirse a partir del dato de cómo solo un $1 \%$ de las ochenta mil transnacionales del mundo ha establecido tales políticas (Vives, 2014, p. 35). Sin embargo, la problemática se vuelve todavía más compleja cuando se consideran otros dos deberes que tienen las empresas con respecto a los derechos humanos: la protección y la promoción, o realización efectiva. Esta difícil, pero irrenunciable tarea, exige a las empresas la puesta en práctica de una visión proactiva y virtuosa de la responsabilidad social (Tangarife-Pedraza, 2008), tanto en el establecimiento de políticas dentro de la empresa, como al involucrar a la organización en los discursos que reflejan e intentan resolver las inquietudes de la esfera pública, en relación con la protección y realización de los derechos humanos (Ibáñez \& 
Ordoñez, 2014). Todo lo anterior debería quedar asentado, al menos a nivel de principio, en los documentos fundamentales de la organización, con el propósito de dotarlos de contenido ético y hacerlos realmente ejecutables dentro y fuera de la empresa.

\section{Los derechos humanos como demandas legítimas de los grupos de interés}

La primera referencia que se tiene sobre los grupos de interés es la clásica obra Strategic Management: A Stakeholder Approach, en la cual su autor, Richard Edward Freeman, definía lo que en los años posteriores se comprendería como stakeholders o grupo de interés, planteándolo como: "Cualquier grupo o individuo que puede afectar o ser afectado por el logro de los objetivos de una empresa" (Freeman, 1984, p. 24).

Algo distintivo de la propuesta de Freeman es que visualiza a la empresa como una red de relaciones, las cuales no solo inciden en los caracteres sociales y jurídicos, sino también en los morales, ya que la relación de los grupos o individuos con la organización promueven una estructuración de valores sobre lo que para ambos resulta correcto o incorrecto en torno a sus decisiones y acciones (Ribeiro \& Ferreira, 2003). Además, Freeman deja muy claro cómo los grupos de interés tienen demandas que deben ser consideradas por la empresa - lo que la lleva a ser responsable hacia ellos-, por todo lo que pudiera relacionarse con su objetivo o misión organizacional (Rodríguez, Moreno \& Vázquez, 2016).

García-Marzá (2007) plantea que esta necesaria relación entre los grupos de interés y la empresa, obliga a que se constituya un diálogo en el que se incluya a todos los afectados por las decisiones de la organización, con el fin de entablar canales de comunicación efectivos que permitan establecer los intereses y valores que legítimamente demandan cumplimiento entre ambas partes. Al determinar lo anterior, la empresa se encuentra en posibilidad de proponer y diseñar estrategias que traten de satisfacer y apegarse a dichos valores, los cuales pueden resultar desde simples acciones específicas, hasta modificaciones estructurales más profundas. Por consiguiente, las empresas deben ser capaces de identificar adecuadamente a todos sus grupos de interés, y tomar en cuenta su capacidad de entablar un diálogo con la organización, las expectativas que estos tienen y las legítimas demandas que los individuos consideren relevantes (Raufflet, Lozano, Barrera \& García, 2012).

Según Cortina, Conill, Domingo \& GarcíaMarzá (2000), la identificación de los grupos de interés debe incluir necesariamente un diálogo que se constituya a partir de la comunicación y la transparencia, fundamentándose en un modelo ético y normativo que conciba a la organización como un ente integrativo. La consecuencia de esto es poder no solo identificar a los grupos y conocer sus intereses, sino también, clasificarlos por sus intereses en particulares, grupales y universales. Además, los grupos no serán considerados únicamente como afectados, sino también como interlocutores válidos que pueden influir en las decisiones y actividades de la compañía. Por otra parte, el diálogo con los grupos de interés permite generar entendimiento y consenso en cuanto a sus intereses universales y consiguir identificarlos, adoptarlos y desarrollarlos como una parte integral de los objetivos organizacionales (Niño de Guzmán, 2015).

Diría Habermas (2000) que el proceso de diálogo es una exigencia para la resolución y generación de estrategias, lo que obliga a un principio de universalización por el cual se establece que una decisión es moralmente correcta cuando puede o podría ser aceptada por todos los afectados, presentes o futuros. De esta forma, la identificación y comunicación con los grupos de interés permite buscar consensos acerca de las normas y procedimientos empresariales que respondan a intereses universales, como, por ejemplo, la protección de sus derechos humanos, los cuales deben marcar la dirección organizacional, así como todas las acciones que se realicen por parte de la empresa.

Por todo lo anterior, el presente artículo plantea la necesidad de adoptar un modelo de identificación de los grupos de interés desde una perspectiva de derechos humanos, el cual apueste por una mejor integración de las demandas legítimas y los intereses universales de estos grupos, en busca de alcanzar una mayor congruencia entre las políticas y acciones de la organización y su compromiso con aportar al desarrollo de estos grupos. 


\section{Propuesta de identificación de los grupos de interés y sus legítimas demandas desde una perspectiva de derechos humanos}

La adopción de una perspectiva de derechos humanos dentro de una organización exige la identificación de los grupos de interés de la empresa, así como la consideración de sus legítimos intereses. Para determinar cómo debe proceder la empresa en relación con sus grupos de interés, se retoma la propuesta de los Procesos de Adecuación Ética de las Organizaciones (PAES), de de la Cruz \& Sasia (2011), quienes han señalado que la perspectiva de gestión de stakeholders debe complementarse con un enfoque de derechos humanos, lo cual permite dar un sentido verdaderamente ético a la relación que la empresa guarda, tanto con sus grupos de interés, como con la sociedad en general.

De la Cruz \& Sasia señalan cómo, desde el punto de vista ético, abordar la relación con los grupos de interés con un enfoque de derechos humanos tiene múltiples consecuencias. En primer lugar, permite superar una visión meramente estratégica en relación con ellos, como la que plantea el modelo de Mitchell, Agle \& Wood (1997); asimismo, proyecta a la empresa más allá del marco legal, ya que le permite determinar qué quiere y a qué se obliga voluntariamente, pero con referentes que impiden que lo "voluntario" se confunda con "arbitrario" o "unilateral". Adicionalmente, pone a la empresa en contacto con otras expectativas sociales que la convocan a la colaboración con otros agentes sociales, y así darle cumplimiento, por ejemplo, a los Objetivos de Desarrollo Sostenible. Por último, permite a la empresa superar el paternalismo o el asistencialismo, de manera que está en capacidad de comprender su acción social en términos de justicia (de la Cruz \& Sasia, 2011).

Aunque ya se ha planteado que los derechos humanos deberían ser referentes normativos para la conducta de las empresas, no se propone una manera de materializar esta exigencia en las operaciones empresariales, lo cual sí aporta la metodología de de la Cruz \& Sasia (2011) para su propuesta de los PAES. Sin embargo, lo que aquí se propone se separa un poco de la propuesta de estos autores, en cuanto a la forma de vincular derechos-deberes y grupos de interés. Ellos señalan que (figura 1), "una manera posible de afrontar esta tarea de una forma sistemática es recorrer el conjunto de derechos que las diversas legislaciones y acuerdos internacionales van cristalizando como el marco que configura una sociedad justa" (de la Cruz \& Sasia, 2011, p. 41), a fin de identificar a los "colectivos (...) a partir del equilibrio derechos-deberes que la organización establece con ellos" (de la Cruz \& Sasia, 2011, pp. 41-42).

Algunos de los instrumentos internacionales mínimos que han de guiar la conducta de las empresas con respecto a los derechos humanos, son: la Carta Internacional de los Derechos Humanos (que incluye la Declaración Universal de Derechos Humanos, el Pacto Internacional de Derechos Civiles y Políticos, y el Pacto Internacional de Derechos Económicos, Sociales y Culturales), así como los principios relativos a los derechos establecidos en la Declaración de la Organización Internacional del Trabajo, referente a los principios y derechos fundamentales en el trabajo (Ramasastry, 2015, p. 243; Ruggie, 2011, p. 16;). Al corpus citado, de la Cruz y Sasia añaden la Carta de Derechos Humanos Emergentes, cuya primera versión fue redactada en el 2004, en Barcelona, durante el Fórum Universal de las Culturas. Esta carta "se inspira en el espíritu y principios de la carta de Derechos Humanos de 1948, pero se construye desde las diversas experiencias y luchas de la sociedad civil global" (de la Cruz \& Sasia, 2011). Además, se deben incluir todas las regulaciones que los organismos nacionales postulen para las organizaciones que laboren dentro de los países.

FIGURA 1. Metodología de de la Cruz y Sasia

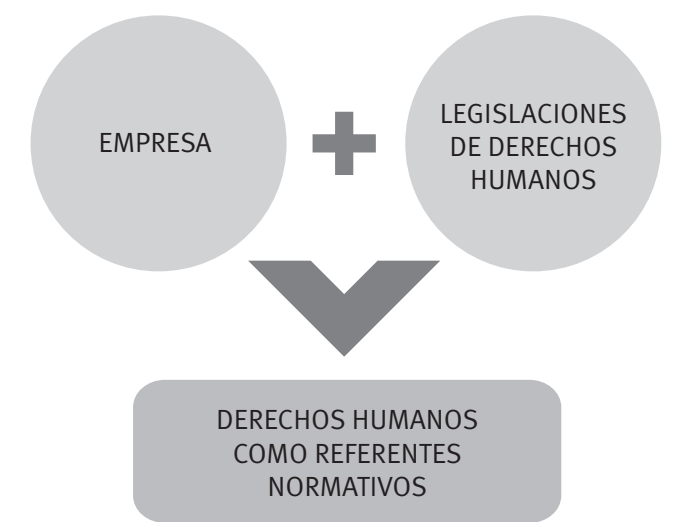

Fuente: elaboración propia a partir de de la Cruz, C., \& Sasia, P. M. (2011). Metodología de adecuación ética y orientaciones para su aplicación en empresas y organizaciones. Bilbao: Universidad de Deusto.

Lamentablemente, este proceso de recolección de normas suele volverse muy complejo para las empresas, sobre todo en las pequeñas y medianas, ya que las obliga a buscar en el gran cúmulo 
de legislaciones que, en ocasiones, no resultan apropiadas para el tipo o giro de actividad organizacional que realizan. Por ello, la mayoría de las pymes pueden terminar fracasando en este proceso de identificación, al adoptar propuestas genéricas que no se relacionan de manera óptima con sus grupos de interés, o al contar con un bajo nivel de apropiación de la práctica de RSE (Moreno \& Graterol, 2011). Lo usual es que se adhieran solo al respeto de la normatividad nacional y que se incluya a los grupos de interés más próximos, sin tomar en consideración la demanda de promoción y contribución de los derechos humanos.

La propuesta que se plantea en este artículo (figura 2) se basa en la idea según la cual este proceso puede ser más sencillo para las empresas, sobre todo para las pymes, si se procede de otra forma. Primero, al identificar a los grupos de interés con los que la empresa entra en relación por el tipo y alcance de su actividad; después (paso 2), la empresa considera, para cada uno de sus grupos de interés, cuáles son los derechos humanos que está obligada a no violar, a proteger, a promover y a contribuir en su realización, ya que las empresas no pueden conformarse con el cumplimiento del umbral mínimo de sus obligaciones con respecto a los derechos humanos (que sería no violarlos), sino que deben ir más allá y así contribuir a su protección, promoción y realización.

Sin embargo, para que la tarea esté completa, la empresa, como último punto (paso 3), deberá determinar qué prácticas o comportamientos conducen al respeto, la promoción y la realización de estos derechos. En ocasiones, el resultado de este último análisis coincidirá con las prácticas que comúnmente se asocian con el mejor modo de relacionarse con un grupo de interés. No obstante, en otras ocasiones, esta perspectiva, como señalan de la Cruz \& Sasia (2011), permitirá a la empresa comprender que sus obligaciones con los grupos de interés van más allá de las prácticas ordinarias, para emprender un esfuerzo o realizar una actividad que no se considera obligatoria, como, por ejemplo, otorgar becas a hijos de sus empleados a fin de que puedan hacer realidad su derecho a la educación.

La determinación de sus grupos de interés, sus derechos y las prácticas que debe implementar en

FIGURA 2. Modelo de identificación de los grupos de interés y sus legítimas demandas desde una perspectiva de derechos humanos

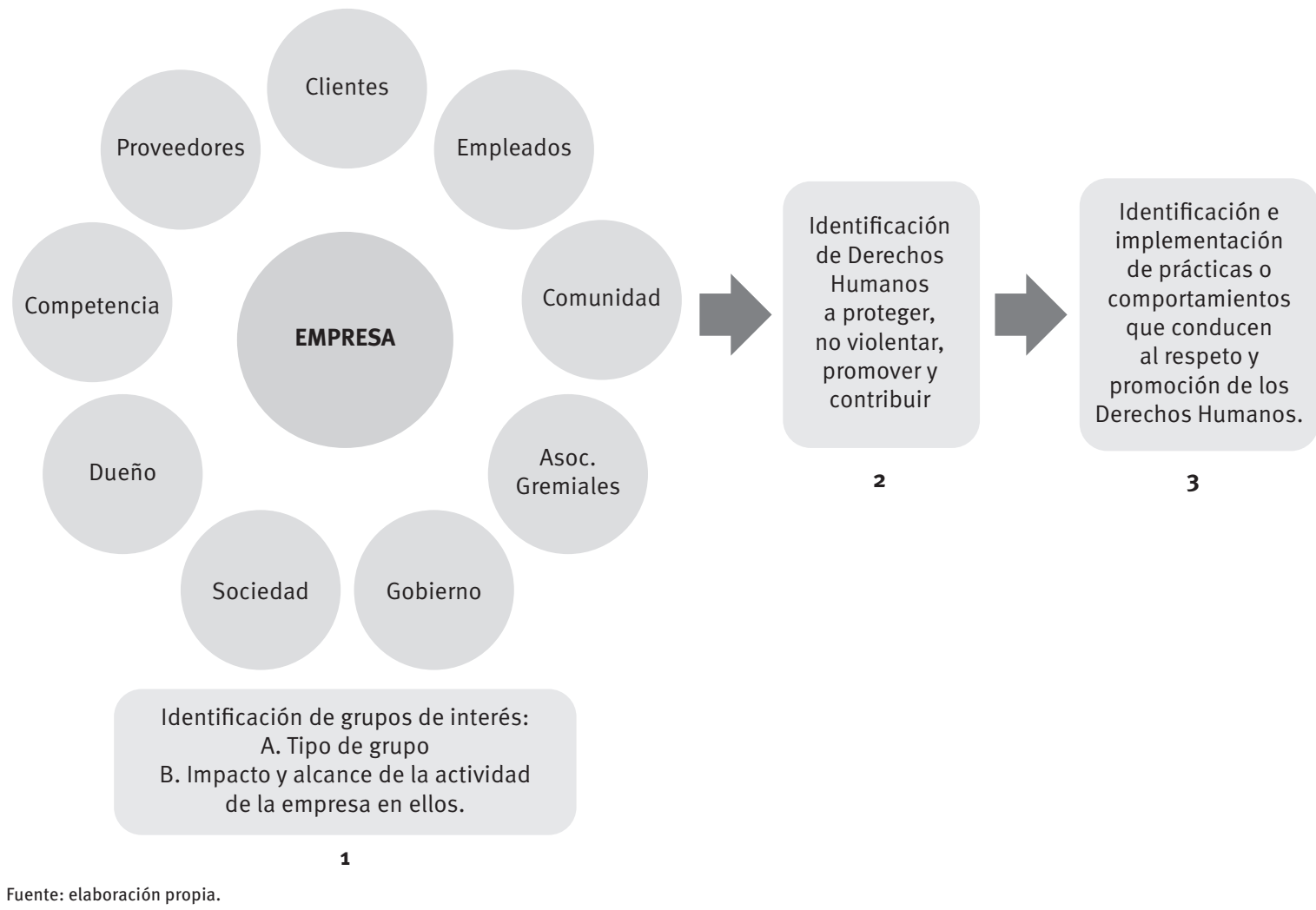


relación con ellos, permitirá a la empresa comprender su actividad como una tarea que se realiza en función del bien común de la sociedad, desde su particular papel de agente económico y distributivo, sin olvidar su capacidad de actuar también en la esfera pública y en la política. Lo anterior le permite contribuir a dar respuesta a la difícil pregunta de cuál es la obligación de las empresas con respecto a los derechos humanos, más allá de la obligación que todas ellas tienen de no violarlos o de reparar toda violación. Esto en razón a que en la determinación de sus obligaciones con relación a los derechos humanos de sus grupos de interés, las empresas podrán identificar aquellas conductas que deben poner en práctica para respetar y realizar los derechos humanos -al menos de sus partes interesadas-.

En algunos casos, el contexto de la operación empresarial es el que puede dar la clave de cuáles son los derechos humanos específicos que debe respetar y realizar. En el caso específico de una empresa de agroindustria cuyo grupo de interés sea una comunidad indígena, en su relación tendrá que tener en cuenta los derechos humanos específicos de ese grupo étnico. La Comisión Europea, en un folleto titulado Mi empresa y los derechos humanos, presenta una lista de derechos humanos dirigida a pymes, el cual puede ser un buen punto de referencia para el momento del proceso que se está comentando (Global CSR \& BBI International, 2012).

Dado todo lo anterior, se reitera que situar los derechos humanos como referentes éticos de los proyectos de responsabilidad que tiene la empresa con sus grupos de interés, aunque resulta ser una excelente forma de vincularse con estos y presenta la ventaja de colocar a la organización en un empeño verdaderamente ético, requiere un esfuerzo extra por parte de las empresas, ya que la adopción de esta medida no puede reducirse a actividades meramente estratégicas o de relaciones públicas. También es importante recordar que dicho esfuerzo debe referirse a todos los derechos humanos de los grupos de interés de la empresa, y no se agota en los derechos laborales y su protección, sino que también abarca los derechos enunciados por el corpus que se ha citado antes, así como su promoción y las labores que contribuyan a la realización de tales derechos.

\section{Resultados}

\section{Resultados en materia de derechos humanos de pymes mexicanas sumadas al Pacto Mundial}

El Pacto Mundial representa un claro intento internacional por fortalecer las relaciones entre los gobiernos, la ciudadanía y las organizaciones para contribuir a resolver los problemas que aquejan a la sociedad (Cantú, 2015). Las líneas de acción se enfocan en temáticas relacionadas con los derechos humanos, las prácticas laborales responsables, el cuidado medioambiental y el desarrollo de sociedades más justas (Duque \& Vargas, 2014). Actualmente, existen más de 12000 firmantes en 170 países, entre los cuales se encuentran, tanto naciones desarrolladas, como en desarrollo, representando así organizaciones de casi todos los sectores y tamaños (ONU, 2017).

En países latinoamericanos como México, el Pacto Mundial ha impactado claramente las prácticas empresariales de la región, pues se ha constituido como una de las redes más grandes de América y la tercera más grande del mundo, con casi 800 empresas, de las cuales el $59 \%$ corresponden al sector privado, y el $41 \%$ al sector público o de la sociedad civil (UNGlobal-Compact, 2015). Como se señaló con anterioridad, generar e implementar acciones ligadas al pacto puede resultar especialmente complejo para las pequeñas y medianas empresas, ya que estas suelen no contar con la capacidad física que les permita llevar a cabo un proceso complejo de aplicación y revisión de lo realizado. Por ende, y para los fines del presente texto y su propuesta, este busca realizar un análisis sobre las pequeñas y medianas empresas mexicanas que se encuentran registradas en la página del Pacto Mundial, con un enfonque en aquellas que cuentan con reportes de progreso actualizados sobre los compromisos pactados en su carta de adhesión.

La vinculación entre el modelo presentado y la revisión de los reportes del Pacto Mundial se centra en mostrar cómo las pymes suelen, en la mayoría de los casos, no alcanzar el nivel adecuado de atención de las demandas legítimas de sus grupos de interés, de modo que solo quedan en proyectos que se centran en el cumplimiento y no en la promoción de sus derechos. La intención de esta revisión es justificar la relevancia de contar 
con un modelo de identificación de los grupos de interés y sus legítimas demandas desde una perspectiva de derechos humanos, con el fin de que, de esta forma, las pequeñas y medianas empresas puedan dar un paso más en la generación de propuestas que integren verdaderamente las demandas de sus grupos. Este análisis se realiza con fines argumentativos, y no como un proceso de validación metodológica del modelo propuesto.

La conformación de la población seleccionada se ha dado a partir del análisis que se presenta en la tabla 1.

TABLA 1. Análisis de situación de pymes en el Pacto Mundial

\begin{tabular}{|c|c|}
\hline $\begin{array}{l}\text { Pequeñas o medianas empresas en el Pacto } \\
\text { Mundial (abril 2017) }\end{array}$ & 318 \\
\hline Con reportes de avance & 194 \\
\hline Sin reportes de avances & 124 \\
\hline Con reportes actualizados & 108 \\
\hline Con cartas de gracia y reportes desactualizados & 86 \\
\hline
\end{tabular}

Con base en los datos de la tabla 1, se realizó un análisis de los 108 reportes actualizados dados de alta ante el Pacto Mundial, con el fin de clasificar los compromisos realizados por estas pymes mexicanas en materia de derechos humanos. Esto al tomar en cuenta si lo realizado se enfoca en proteger, no violentar, promover o contribuir a esta materia. De los reportes analizados, los resultados se presentan en la tabla 2 .

TABLA 2. Clasificación de las acciones en materia de derechos humanos en pymes mexicanas inscritas al Pacto Mundial

\begin{tabular}{lc}
\hline Incluyen temas de los derechos humanos & 108 \\
\hline No violentan los derechos humanos & 108 \\
\hline $\begin{array}{l}\text { Promueven y contribuyen al desarrollo de los } \\
\text { derechos humanos }\end{array}$ & 36 \\
\hline
\end{tabular}

Fuente: elaboración propia con base en ONU. (2017, abril). Global Compact-Our Participants. Recuperado de https://www.unglobalcompact.org/what-is-gc/ participants

De los 108 reportes analizados, la totalidad de ellos plantean políticas o acciones con el fin de prevenir, proteger y evitar todo tipo de acciones que violentarían los derechos humanos. Este tipo de acciones incluye la adhesión a los parámetros que exige la ley nacional, políticas antidiscriminación, la generación de códigos de ética e, incluso, la constitución de áreas especializadas con miras a mejorar la comunicación con los grupos de interés y así conocer sus necesidades.

Cabe señalar que solo 36 de las 108 organizaciones han conseguido dar un paso más allá, al contemplar, dentro de sus reportes de avances, acciones que buscan promover o contribuir al desarrollo de los derechos humanos de sus grupos de interés, y no simplemente enfocarse en aspectos que legalmente ya deberían contemplar. Algunos ejemplos de estas acciones son:

- La participación de los colaboradores de la empresa en eventos o foros donde se promocione la sostenibilidad laboral como sistema de gestión de negocios (Promotora ACCSE S. A. de C. V.).

- Actividades de formación sobre políticas y procedimientos relativos a los aspectos de derechos humanos relevantes para las operaciones de la organización, así como capacitación formal específica sobre la materia y su aplicación (Construcciones Nirvana S. A. de C. V.).

- Planes de trabajo conjunto con organismos estatales y civiles que fomentan los derechos humanos, así como charlas a sus colaboradores de organismos oficiales como la Comisión Nacional de Derechos Humanos (Agrícola Chaparral S. P. R. de R. L.).

- Generación de organismos internos y canales de comunicación autónomos a la empresa que faciliten la comunicación y honestidad sobre prácticas o situaciones que afecten los derechos humanos (Etisa de Guadalajara, S. A. de C. V.).

- Proyectos de inclusión de grupos vulnerables en procesos internos de la organización (BCYSA, Servicios Industriales, S. A. de C. V.).

- Generación de trípticos y material informativo sobre temas de interés que se relacionan con derechos humanos para los colaboradores y sus familias (CAMINEC, S. A. de C. V.).

Lamentablemente, la mayor parte de estas políticas, aunque efectivamente van más allá de las cuestiones simplemente legales, de respeto y no violentación, son todavía acciones que, más que contribuir al desarrollo de los derechos humanos, se quedan simplemente en una labor de sensibilización, lo cual resulta insuficiente si lo que realmente pretenden las empresas es responder a las demandas legítimas de sus grupos de 
interés. Esta es otra de las razones por las cuales es necesario que las pymes cuenten con un modelo que les permita conocer los compromisos reales que tienen hacia la sociedad en el tema de derechos humanos, ya que al no contar con claridad sobre sus posibles obligaciones y sus grupos de interés, aunque la empresa cuente con un modelo de negocio responsable y sostenible, sus acciones son superficiales y sin un verdadero impacto en el exterior de la organización (Briseño, Lavín, \& García, 2011).

Como ya se ha señalado con anterioridad, el que las empresas busquen proteger y no violentar los derechos humanos resulta ser una acción plausible, pero esto no resulta congruente si lo que se pretende es demostrar un verdadero interés por el desarrollo de sus grupos. Sin embargo, y de manera especial para las pymes, este tipo de decisiones no es una tarea sencilla, ya que implica la existencia de una capacidad organizacional lo suficientemente significativa para generar proyectos que vayan más allá de sus labores y procesos cotidianos. Por ende, contar con un modelo como el propuesto resulta muy conveniente para este tipo de empresas, pues facilitaría considerablemente el proceso de identificación de sus grupos de interés y sus legítimas demandas, al ser este el primer paso en el propósito de generar acciones verdaderamente efectivas que promuevan acciones de responsabilidad organizacional en materia de derechos humanos.

\section{Conclusiones}

Si bien proponer un modelo de identificación de los grupos de interés y sus legítimas demandas desde una perspectiva de derechos humanos parece ser una excelente oportunidad para que la empresa se promueva como una organización realmente comprometida con los intereses de sus grupos, esto no quiere decir que sea fácil para una pyme llevar a cabo el procedimiento planteado, pues, aunque contar con la información que pretende el modelo resulta ya ser algo valioso, no es suficiente para generar un verdadero cambio en la compañía. Por tanto, es posible cuestionar que una organización, sobre todo si esta es pequeña o mediana, de manera individual y sin ayuda, esté en capacidad de identificar áreas de oportunidad con las que se mejore su relación con los grupos de interés, ya que desde una percepción cotidiana, por lo general, las cosas funcionan adecuadamente tal como se llevan a cabo.

Así como se da en los procesos de certificación o mejoramiento de la calidad de los productos o servicios, el fomento de una relación que responda a los intereses legítimos de los grupos de interés resulta ser una parte fundamental en la manera en que la empresa responde a sus obligaciones con el entorno y la sociedad (Sotelo \& Durán, 2014). Al final de cuentas, ¿qué es una empresa, sino un ente dedicado a generar valor al satisfacer las necesidades y demandas de la sociedad y de sus miembros? Por ende, el presente modelo le permite acercarse un paso más a la consecución de su razón de ser, y enfocarse en la necesidad de que dicho valor sea compartido por todos sus grupos de interes, cuestión que embona con la noción básica de la responsabilidad social empresarial.

Los ejemplos analizados de pymes mexicanas son un factor que, más que validar, nos da razones por las que un modelo como el propuesto resulta adecuado y útil para las empresas, ya que les permitiría, al identificar las legítimas demandas de sus grupos de interés, hacer propuestas más integrales de promoción de sus derechos, y no solo de su no violentación.

Acciones como las que promueve Cosméticos Natura S. A., organización que no solo protege los derechos de los grupos vulnerables, sino que además integra a su plantilla laboral a miles de mujeres e indígenas de Brasil, o las políticas de selección de proveedores de la Procesadora Nacional de Alimentos C. A. (Pronaca), la cual, además del nivel táctico y operativo, toma en cuenta un enfoque estratégico dentro del cual se incluyen criterios de derechos humanos, prácticas laborales y relación comunitaria, o incluso Alpina, empresa alimentaria que ha incluido a grupos vulnerables colombianos dentro de sus canales de distribución con el fin de mejorar su calidad de vida, son claros ejemplos de cómo, más allá de la no violentación, hay mucho que aún se puede hacer por parte de las empresas en temas de derechos humanos.

La protección, no violentación, promoción y contribución a los derechos humanos es una responsabilidad a la que todos los miembros de la sociedad deben sumarse, ya que si algo no se puede negar es que toda empresa u organización, sea grande, mediana, pequeña o micro, se encuentra constituida por personas con intereses, deseos y objetivos que pretenden alcanzar. Así, a fin de que 
esto suceda, se les debe tratar con la dignidad y el respeto que merecen, por el simple hecho de ser seres humanos.

\section{REFERENCIAS}

Alfonso, M. (2013). Empresas y derechos humanos: una responsabilidad en construcción. Revista Facultad de Derecho y Ciencias Políticas, 43(118), 349-385.

Briseño, A., Lavín, J., \& García, F. (2011). Análisis exploratorio de la responsabilidad social empresarial y su dicotomía en las actividades sociales y ambientales de la empresa. Contaduría y Administración, 233, 79-90.

Calderón, G., Murillo, S., \& Torres, K. (2003). Cultura organizacional y bienestar laboral. Cuadernos de Administración, 16(25), 109-137.

Cantú, H. (2013). Empresas y derechos humanos: ¿Hacia una regulación jurídica efectiva o el mantenimiento del status quo? Anuario Mexicano de Derecho Internacional, 13, 313-354.

Cantú, H. (2015). La OCDE y los derechos humanos: el caso de las directrices para empresas multinacionales y los puntos de contacto nacional. Anuario Mexicano de Derecho Internacional, 15, 611-658.

Cortina, A., Conill, J., Domingo, A., \& García-Marzá, D. (2000) Ética de las empresas. Claves para una nueva cultura organizacional. Madrid: Trotta.

De la Cruz, C., \& Sasia, P. M. (2011). Metodología de adecuación ética y orientaciones para su aplicación en empresas y organizaciones. Bilbao: Universidad de Deusto.

Duque, Y., \& Vargas, D. (2014). Una mirada a las prácticas de anticorrupción de las organizaciones adheridas al Pacto Global en Colombia. Revista CS, 13, 145-182.

Freeman, R. E. (1984). Strategic management. A stakeholder approach. Toronto: Pitman.

García-Marzá, D. (2007). La RSE desde la ética empresarial: un Nuevo diseño institucional para las empresas. Fundación Jaime Vera (Ed.), La responsabilidad social de las empresas: Miradas desde la izquierda (pp. 201-226). Madrid: Galapagar.

Global CSR, \& BBI International. (2012). My business and human rights: a guide to human rights for small and medium-sized enterprises. Bruselas: Comisión Europea.

Habermas, J. (2000). Aclaraciones a la ética del discurso. Madrid: Trotta.

Ibáñez, P., \& Ordoñez, V. (2014). Papel de las empresas y de los estados en la debida diligencia en derechos humanos. International Law: Revista Colombiana de Derecho Internacional, 24, 219-246.
Ki-moon, B. (2016, enero 20). El sector privado es importante para cumplir los ODS. Recuperado de http://www.unmultimedia.org/radio/spanish/2016/01/el-sector-privado-es-importante-para-cumplir-los-ods-dice-ban-ki-moon/\#.VzN6IPnhDIU

Mitchell, R. K., Agle, B. R., \& Wood, D. J. (1997). Toward a theory of stakeholder identification and salience: defining the principle of who and what really counts. Academy of Management Review, 22(4), 853-886.

Moreno, Z., \& Graterol, D. (2011). Prácticas de responsabilidad social empresarial en la pyme. Un estudio en el sector metalmecánico de Barquisimeto, Estado Lara, Venezuela. Contaduría y Administración, 235, 175-194.

Mota, M., Castelo, D., \& da Silva, I. (2015). Compromisso com a RSE no Pacto Global da Organizacao das Nacoes Unidas. INNOVAR. Revista de Ciencias Administrativas y Sociales, 25(58), 81-90.

Niño de Guzmán, J. (2015). La cultura organizacional en el contexto de la globalización. Apuntes Universitarios, 5(2), 19-40.

Pacto-Mundial. (2015). Los 10 principios del Pacto Mundial. Red Pacto Mundial México. Recuperado de http://www.suplementopactomundial. com/2012/09/los-10-principios-de-pacto-mundial/

Pedroza, S. (2007). Derechos humanos en el trabajo. Revista Latinoamericana de Derecho Social, 4, 267-270.

Peláez, J. (2015). Legislación minera y derechos humanos: el derecho en la encrucijada. El Cotidiano, 194, 107-120.

Ramasastry, A. (2015). Corporate social responsibility versus business and human rights: bridging the gap between responsibility and accountability. Journal of Human Rights, 14(2), 237-259.

Raufflet, E., Lozano, J.-F., Barrera, E., \& García, C. (2012). Responsabilidad social empresarial. México: Pearson.

Ribeiro, É., \& Ferreira, M. (2003). Cultura e comprometimento organizacional em empresas hoteleiras. RAC-Revista de Administracao Contemporanea, 7, 205-222.

Rodríguez, A., Moreno, M., \& Vázquez, E. (2016). Responsabilidad social empresarial como factor de sustentabilidad para el mejoramiento del capital humano en las empresas mexicanas. XXI Congreso Internacional de Contaduría, Administración e Informática. Ciudad de México: Universidad Nacional Autónoma de México

Ruggie, J. (2011). Principios rectores sobre las empresas y los derechos humanos: puesta en práctica del marco de las Naciones Unidas para "proteger, respetar y remediar". Reporte del Representante Especial del Secretario General para la cuestión de 
los derechos humanos y las empresas transnacionales y otras empresas (A/HRC/17/31). Nueva York: ONU. Recuperado de www2.ohchr.org/SPdocs/ Business/A-HRC-17-31_sp.doc

Sotelo, J., \& Durán, M. (2014). La responsabilidad social empresarial en empresas de la ciudad de León Gto., y su compromiso con la comunidad y el medio ambiente. XIX Congreso de Contaduría Administración e Informática. Ciudad de México: Universidad Nacional Autónoma de México

Tangarife-Pedraza, M. (2008). De la responsabilidad de la empresa y los derechos humanos. International Law. Revista Colombiana de Derechos Internacional, 12, 145-182.

Trejo, L. (2015, junio 1). El Pacto Mundial: 10 años en México. El Economista. Recuperado de http:// eleconomista.com.mx/industrias/2015/06/01/ pacto-mundial-10-anos-mexico.
ONU. (2017, abril). Global compact-Our Participants. Recuperado de https://www.unglobalcompact. org/what-is-gc/participants

UNGlobal-Compact. (2015). Pacto Mundial. Red Pacto Mundial. Recuperado de http://www.pactomundial.org.mx/site/

Urteaga, E. (2008). El debate internacional sobre el desarrollo sostenible. Investigaciones Geográficas, 46, 127-137.

Vives, A. (2014). Derechos humanos para empresas normales y corrientes. En Fundación Étnor (Ed.), ¿Para qué sirve realmente la ética de la empresa? XIII seminario permanente de Ética Económica y Empresarial (pp. 25-43). Valencia: Fundación Étnor. 